\title{
“La Investigación Pedagógica: formas y posibilidades que se entretejen en la configuración de la experiencia singular del ser y quehacer del maestro"
}

\section{The Pedagogical Research: ways and possibilities that interweave in shaping of the singular experience of teacher's being and life's work"}

\author{
Por: Claudia Janneth Jaramillo Sánchez ${ }^{1}$
}

"Es difícil saber si el mundo en que vivimos es sueño o realidad"

\begin{tabular}{|c|c|c|}
\hline & \multirow[t]{2}{*}{ Kim Ki-duk } & Domidh 2400 001 \\
\hline & & 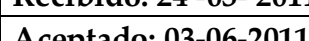 \\
\hline
\end{tabular}

El presente escrito pretende mostrar algunas formas y posibilidades que aluden al reconocimiento de la investigación pedagógica como un campo de creación constante, como eje de pasión y conmoción que le permite al maestro inquietarse por construir y deconstruir aquello que es sabido y permanece inamovible, a partir de múltiples miradas en torno a su experiencia de vida singular como sujeto ético, político, cultural y estético. Así mismo, este escrito contempla acercamientos de los maestros en formación y en ejercicio, a la investigación, así como hacia algunas relaciones que se empiezan a tejer tanto desde aquellas posibilidades que afloran en su nombre, como aquellas que la hacen permanecer invisible. En este sentido, se exalta la configuración del ser y quehacer del maestro y de la investigación como posibilidad de movilizar el pensamiento y el sentimiento desde los lentes de la pedagogía que aluden a la creación de otros hilos que constituyan otros mundos posibles en relación con el maestro, los sujetos, los escenarios y las dinámicas que se configuran.

De ahí que, a lo largo del escrito se pretende plasmar la inquietud por el maestro de Biología y aquellas posibilidades que emanan y se inspiran en la investigación pedagógica, a propósito de repensar las formas en que los maestros estamos tejiendo relaciones y comprensiones sobre lo vivo, en tanto se trata de vislumbrar posibilidades que a partir del despliegue del pensamiento y la creación configuren el reconocimiento de lo vivo y sus relaciones con la vida desde otras ópticas.

Las fibras a partir de las cuales se desarrollan e hilan las ideas de este escrito, emanan de mi experiencia singular en torno a la investigación y sus múltiples lugares de constituirse y comprenderse en el Departamento de Biología, así como los escenarios que éste mismo ha posibilitado durante mi formación: componentes, práctica pedagógica, línea de investigación, y desde luego, los sujetos que transitan y se movilizan por estos escenarios, sujetos con los que ha sido posible crear, pensar, imaginar, discutir y sin duda movilizarme desde la opción de hablar, escribir, leer y escuchar al otro.

Las consideraciones finales que emergen de esta reflexión no buscan interpolar acuerdos sobre el perfil del maestro, la investigación pedagógica o la Biología, por el contrario, pretenden abrir un espacio que permita repensar estas categorías y sus posibles interrelaciones desde la experiencia singular de cada maestro, generando inquietud, desconcierto y conmoción. No se trata pues de

${ }^{1}$ Estudiante de Licenciatura en Biología. Universidad Pedagógica Nacional. claxa414@gmail.com 
encasillar la mirada a través de estas líneas, sino de ampliarla en tanto es posible recomponer las aristas que nos concatenan en cada paso.

Palabras Clave: Investigación Pedagógica, Maestro, Biología, Experiencia, Inquietud.

\section{Abstract}

This paper expects to show some ways and possibilities that mention the recognition of the pedagogical research like a field of constant creation, as an axis of passion and commotion that allows the teacher to worry for constructing and deconstructing what is known and remains immovable, through many looks around his singular life experience as an ethic, politic, cultural and esthetic individual. Likewise, this paper consider rapprochements of the undergraduate students and teachers to research, as well as some relations that begin to weave as from these possibilities that surface in its name, as these that make them stay invisible. In this sense, not only teacher's being and task are praised but also research as a possibility of mobilizing thoughts and feelings from the pedagogy lens that mention to the creation of other threads that constitute other possible worlds in relation to the teacher, individuals, scenarios and dynamics that make it up.

That's the reason why, this paper expects to capture the concern about the biology teacher and those possibilities that emanate and are inspired by pedagogical research, to rethink the ways in which teachers are weaving relations and understandings about the living, while it deals to glimpse possibilities that from the display of the thinking and the creation configure the recognition of the living and their relations with the life from other points of view.

The fibers through which the ideas of this document are developed and linked, emanate from my singular experience in research and its several places of constituting and been understood in the Biology Department, as well as the scenarios that it has provided during my formation: subjects, pedagogical practicum, the research line, and of course, the subjects that travel and mobilize through these scenarios, subjects with whom it has been possible to create, think, imagine, discuss and undoubtedly mobilize myself from the possibility of speaking, writing, reading and listening to the another person.

The final considerations that emerge from this reflection don't seek to interpolate agreements about the teacher's profile, pedagogical research o Biology; on the other hand, they expect to open a space that allows to rethink these categories and their possible interrelations from the singular experience of each teacher, generating concern, atmosphere of uncertainty and commotion. It's not aimed categorizing the perspective trough these lines, but wide it while it is possible to recompose the awn that concatenate us in each step.

Key Words: Pedagogic research, Teacher, Biology, Experience, concern.

La investigación, es quizá uno de los pilares que más ha fomentado el pensar como acto de creación, y en su paso, ha dado apertura a múltiples posibilidades de expresar la existencia del hombre, sus afanes y necesidades más próximas, en tanto genera inquietud por aquello que no es sabido, no se ha dicho, e incluso, se ha dicho siempre de la misma forma, bajo la misma luz y bajo la misma sombra. La investigación no se detiene, pero en su paso deja muchos rastros y huellas que no solo se traducen en un fin determinado, sino en la experiencia que trae consigo, tanto en los trayectos que se recorren a su paso como en las oscilaciones que este mismo trayecto va generando. Sin embargo, es claro que la inquietud no cesa, por el contrario, permanece latente y perdura como algo recurrente, la inquietud entonces, se halla presente en nuestro diario vivir, indistintamente de raza, sexo, credo, religión, condición social o en definitiva de nuestra experiencia de vida, la cual nos invita a preguntarnos por ciertas cosas y no por otras, nos invita a quitarnos el sesgo que le hemos puesto a nuestra mirada cuando nos interrogamos por si 
mismos; de ahí que la investigación emane de la inquietud y brille en su estado naciente aún cuando no se obtenga respuesta alguna, o incluso, aún cuando no se obtengan la respuestas que esperamos encontrar.

Teniendo en cuenta lo anterior, este escrito pretende mostrar algunas formas y posibilidades que se entretejen en la configuración de la investigación pedagógica como campo de creación y emancipación del pensar y sentir constante, que proporciona otras miradas de los acontecimientos y las experiencias que se constituyen en su ir y devenir. Por ello, se inspira en mi experiencia singular como investigadora y los trayectos que he recorrido a lo largo de mi configuración como maestra, en tanto la posibilidad de movilización de mi pensamiento, una movilización dada por los acontecimientos que fuerzan a pensar más allá de la misma voluntad, acontecimientos que han generado conmociones, afectos y rupturas en torno a la investigación desde los diferentes escenarios y espacios que posibilita el Proyecto Curricular de Licenciatura en Biología (PCLB) tales como, el ciclo de fundamentación, la práctica pedagógica y las líneas de investigación, escenarios gratificantes en los que se han involucrado sujetos y estilos de vida propios que se han encargado de matizar este escrito desde la singularidad. En ese sentido, se exalta el ser y quehacer del maestro, sus posibilidades de crearse y recrearse dentro de la investigación, las dinámicas y escenarios que lo constituyen entre la sinergia de lo visible y lo invisible, así como algunas prácticas que tienden a desdibujar su rastro y huella por los escenarios donde transita.

La investigación pedagógica a propósito de las palabras del maestro Martínez Boom (2010, abril), implica hacer un esfuerzo por tratar de "pensar diferente y decir aquello que es sabido destituyendo el lugar común" en este sentido, la esencia de la investigación no se condensa a través de los resultados, sino a través de todo aquello que acontece en su ir y devenir, es decir, aquello que configura el trayecto de la investigación desde las múltiples posibilidades de apasionar, sorprender y producir movilizaciones que se inquietan por mirar lo recurrentemente visible, pero también, lo invisible e intangible y por configurar otros modos de existencia desde la problematización, capaz de producir oscilaciones en los modos como nos relacionamos consigo mismos y con el otro y en ese sentido, afecta nuestros modos de ser y habitar en el mundo. La investigación, interroga y pone en tela de juicio nuestros modos de ser y habitar el mundo, confronta lo que somos sin instaurar "verdades" o quizá legitimar las de otros, con el propósito de transformarnos constantemente no como nuevos sujetos en un nuevo mundo, sino como sujetos creadores de nuevas posibilidades en torno a sus modos de existencia; es así que el maestro como sujeto intelectual crea y recrea constantemente su ser y quehacer pensando lo impensado, pensando de otro modo, diciendo lo que es sabido desde lentes matizados de rupturas y recomposiciones, así como visibilizando lo que permanece invisible.

Así mismo, la investigación como experiencia singular, se vislumbra desde la posibilidad de mostrar otras formas de ser y estar del maestro en su propio quehacer pensado desde la pedagogía, como posibilidad de movilización al ensanchar la mirada hacia otras formas que intenten matizar la creación desde opciones capaces de romper con el paradigma que relega dicho quehacer a los estrechos márgenes de la transmisión del conocimiento, o incluso como el animador y acompañante de la información, como el funcionario que responde a las lógicas contemporáneas del discurso de la calidad en la educación. El ser y quehacer del maestro desde lo planteado por la línea de investigación Trayectos y Aconteceres (2009), trasciende el aula a otros espacios donde se entremezclan las múltiples vicisitudes de los sujetos que merecen la opción de pensar, sentir y reflexionar a partir de diferentes miradas, para que sea cada uno de ellos quien tome opciones y no sean otros quienes lo hagan por ellos.

Por ende, la formación política, ética y estética del maestro atraviesan su ser y su quehacer, desde la posibilidad de debatir y discutir con el otro, con el propósito de sentar una postura critico-reflexiva en torno a lo que se ve, lo que se dice y los modos de decirlo, a propósito del funcionamiento en el que se enmarca aquello que es dicho y por tanto visible. En ese sentido, el maestro como sujeto político, ético y estético se entreteje desde su experiencia singular en relación con el modo en que se conduce por la vida y su estilo propio de pensar, sentir y expresarse en la palabra, la escritura y la kinesia; el maestro es pues, un sujeto capaz de mirarse a sí mismo para construir y deconstruir otras miradas que conlleven a problematizar su ser y quehacer; es un sujeto que contempla la posibilidad de reconocer la existencia de 
otros, y que igualmente propicia que los otros se logren mirar a sí mismos, al pensar y sentir más despacio, al ser sujetos de experiencia, creación y trasformación.

Ahora bien, acudiendo un poco a la perspectiva histórica, la investigación pedagógica ha contribuido a ampliar los senderos del debate, la discusión y la crítica desde diferentes posturas, una de las más reconocidas es el Movimiento Pedagógico Colombiano, en tanto acontecimiento político y cultural que emergió en los años 80, como respuesta a la renovación curricular de la educación, que conllevo a un posicionamiento estratégico de los maestros respecto a la pregunta y la inquietud por sí mismos, por su oficio como intelectual de la pedagogía, en la medida en que se empezaron a desplegar formas colectivas de resistencia, en tanto se configuraban formas de relación con el mundo y con la cultura. (Martínez, 2008)

Tras la constitución de este Movimiento, se gestaron grupos de investigación a su interior tales como "Historia de la Práctica Pedagógica en Colombia", "Enseñanza de las Ciencias" y "El Campo Intelectual de la Educación en Colombia", que impactaron profundamente en la visión de la pedagogía y contribuyeron a fundamentar propuestas innovadoras. Tamayo (2006)

De ahí que la movilización que propició la constitución del Movimiento Pedagógico trajo consigo el fomento de la investigación y por ende el de la inquietud y la interrogación propia, a partir de formas singulares de imbricación en el ser y quehacer del maestro:

"Contamos con una amplia trayectoria investigativa nacional que es necesario recuperar en función de pensar la escuela, el maestro y la educación más allá de los reducidos intereses de los organismos de financiación y cooperación técnica y de quienes desde el estado, se han empeñado en olvidar y borrar de la memoria educativa nacional las discusiones, debates y producción alrededor de la complejidad propia de la tarea educativa y pedagógica" (Noguera, 2002: 98)

"El impulso amorfo del Movimiento Pedagógico mutó a escritura, formas de organización, experiencias de estudio, preguntas investigativas, encuentros regionales y nacionales, conformó equipos y redes, organizó rutas y viajes...una pleyadé de nominativos que gestaron su forma plural y divergente." (Martínez, 2008:11)

Otros aportes importantes del Movimiento Pedagógico a la investigación, según Tamayo (2006) tiene que ver con la consolidación del Centro de Estudios e Investigaciones Docentes (CEID) a nivel nacional que reunió investigadores y dirigentes sindicales en la reflexión sobre los problemas de la educación y la enseñanza. Del mismo modo, publicaciones como la Revista Educación y Cultura constituye una verdadera joya en el campo pedagógico, con más de 70 números, la revista ha sido el mejor aporte en el debate nacional sobre temas como: el movimiento pedagógico, el currículo, la autonomía escolar, la defensa de la educación pública, la ley general de educación, las reformas, la participación política, la formación de los maestros, entre otras. De ahí que la revista se considere uno de los medio que más ha contribuido a la difusión del pensamiento pedagógico nacional en tanto ha sido una revista pensada, dirigida y sostenida por el magisterio; en esta medida, muchos grupos de discusión sobre pedagogía se formaron a raíz de la lectura de la revista.

En esta medida, el Movimiento Pedagógico es un gran referente de las movilizaciones y oscilaciones que ha suscitado la investigación de manera individual y colectiva al pensar la escuela, el maestro y el saber que le es propio: el saber pedagógico, un referente que no debemos descartar a la hora de sentar posición frente a la investigación y con ello, los acontecimientos que nos trastocan. De hecho la investigación se configura como condición de posibilidad en tanto propicia encuentros y deconstrucciones consigo mismos, con los otros y con la escritura.

No obstante, es necesario reconocer que así como se han dado condiciones de posibilidad para ensanchar la mirada de la investigación pedagógica hacia diferentes formas de visibilizarla, también se han dado condiciones de posibilidad para relegarla, restringirla o incluso invisibilizarla; es así como a partir de los planteamientos de Cerda (2003), se evidencia que a mediados del siglo XIX en nuestro país, la depreciación del género llevó a estigmatizar la "enseñanza" y por ende, la investigación pedagógica, ambas 
consideradas como labores acordes al perfil femenino que requerían de poco intelecto puesto que se basaban en la mera "instrucción". Desde entonces, es evidente que preparar para un "saber hacer" a partir de un conocimiento instrumental se ha convertido en un importante objetivo económico, en un discurso con trasfondo ideológico que se intenta justificar como una alternativa de solución propicia que permite el acceso a la "educación", al campo laboral y a la formación permanente, es allí donde se empieza a crear competencia en las bases de los valores de la calidad: la eficacia, la eficiencia y la efectividad, allí, no resulta trascendental pensar en nuestras pasiones o afectos, en aquello que genera inquietud o en poner en cuestión lo establecido.

Por otro lado, la investigación en educación ha sido considerada como un pilar de progreso para la nación y en este sentido, se ajusta a las necesidades que imperan en la actualidad, que tienen mucho que ver con considerarla como una herramienta de cambio ligada a la producción, los resultados y las posibles alternativas de solución en torno a problemáticas ambientales o educativas. De ahí que, se empiezan a definir ciertos parámetros y marcos que los proyectos de investigación deben cumplir para ser apoyados, así como la predilección por temáticas particulares que se hacen urgentes e importantes de visibilizar, hablar y comprender, donde se hace evidente el direccionamiento y los intereses desde los cuales se debe investigar, en este sentido, se desplaza al maestro de su inquietud y su creación, no hay espacio para la singularidad de su experiencia, sus afectos o pasiones, puesto que al instaurarse bajo las lógicas de la productividad, se crean fuerzas, relaciones de poder y saber en las formas de constitución del maestro.

Retomando a Cerda (2003), algunos de los impedimentos más frecuentes que tienden a invisibilizar la investigación pedagógica, giran en torno a la presión, rutina y costumbre a la que se enfrentan muchos maestros, así como su resistencia al cambio, a nuevos horizontes y posibilidades que permitan articular la investigación con su práctica pedagógica e incluso considerarla como parte de su ser y quehacer; esta resistencia, puede darse a causa de un sin número de razones personales y/o colectivas, sin embargo, es la falta de tiempo la más común y la que trae consigo la inmersión de la rutina en el diario vivir del maestro.

En este sentido, Larrosa (2003) plantea algunos hechos interrelacionados entre sí, que en la actualidad no nos deja ver más allá de lo que nos pasa a diario, uno de ellos es precisamente la falta de tiempo, teniendo en cuenta que el ritmo de los sucesos se da velozmente y el exceso de trabajo muestra cómo dichos sucesos se rigen bajo la acción (hacer, producir, modificar algo). Desde esta perspectiva, los avatares del hombre contemporáneo indican que aún cuando acontezca mucho en su diario vivir vinculando un hecho que sucede con otro desde la acción, no pase nada en términos de experiencia, de afectar de algún modo, inscribir marcas y dejar huellas. De ahí que, el maestro al dejar de lado su matiz creador, asuma las funciones del docente y se rija en cierto modo bajo las presiones del tiempo y la consecución de resultados.

Sin embargo, vislumbrar la experiencia más allá de todo lo que acontece a diario como un cúmulo de sucesos, de cierta manera, nos impide hacer un alto en el camino para recomponer nuestra experiencia como un encuentro que permite pensar, mirar, escuchar y sentir más despacio, posibilitando el cruce de diferentes miradas, que no recaen en un cúmulo de sucesos anclados de forma repetitiva sino en acontecimientos que generan rupturas, inquietudes, encuentros y desencuentros consigo mismo; la experiencia entonces, en vez de significar encierro dentro de los propios pensamientos, sentimientos y sensaciones, involucra un intercambio activo y atento frente al mundo así como los acontecimientos que se van configurando en sí mismos. Es por ello que el maestro, va dibujando y desdibujando en su experiencia de vida, trayectos singulares desde los diferentes escenarios en los que se desenvuelve.

Por ello, considero que desde un sentido figurado, la experiencia emerge a partir de la paleta de un artista la cual se compone de diferentes colores y tonalidades que él mismo va tomando a través de pinceladas y trazos de pensamiento, creación, imaginación y sensibilidad, pinceladas y trazos que matizan y transfiguran los trayectos de su vida, los cuales se plasman en improntas con las que el artista va irrumpiendo el mundo y va configurando tanto su ser como su quehacer, este artista al que hago alusión es el maestro, un artista con pensamiento creativo vislumbrado desde las ventanas de escape, el abanico de posibilidades y las luchas que configura entre lo transparente y lo opaco, lo claro y lo oscuro, así como lo 
visto y no visto, donde la visibilidad de la luz y sus formas de luminosidad permiten que el maestro deje de existir como reflejo y destello de una otredad, en tanto se hace posible su recomposición en el profundo pensamiento.

A partir de estas problematizaciones, la posición del maestro como sujeto ético, político y estético frente a las múltiples realidades que configuran los sujetos invita a crear formas y posibilidades desde la investigación pedagógica que propicien la mirada hacia sí mismos como experiencia de vida que trasforma y atraviesa el modo en que habitamos el mundo, de esta manera, es posible desplegar la movilización del pensamiento y la capacidad de fortalecer el sentido del ser mismo desde la reflexión crítica del acto propio, logrando así, construir y deconstruir lo que es sabido, lo que permanece inamovible e inalterable, aquello que tiende a uniformar el pensamiento y a limitar la posibilidad de crear, soñar, imaginar y ser participes de otras miradas.

En este sentido, desde los planteamientos hechos por Zuluaga (2003), es necesario formar maestros de alta capacidad investigativa que propicien diversos espacios basados en la comunicación mutua entre ellos y los diferentes campos de acción tanto social como académica; adicionalmente, la creación de una comunidad intelectual que comprenda investigadores en pedagogía, maestros en formación y maestros en ejercicio, para que de esta manera se fortalezca y potencialice la crítica al interior y por ende, se establezca la diferencia desde diferentes puntos de partida como garantía de supervivencia y enriquecimiento del trabajo intelectual.

De esta manera, la investigación pedagógica como espacio de creación singular del sujeto maestro emana de los trayectos que han configurado su experiencia de vida, es así que parte de esos trayectos devienen desde sus primeros acercamientos a las formas y posibilidades de la investigación; en nuestro caso, desde algunos de los objetivos planteados por el Proyecto Curricular de Licenciatura en Biología (PCLB), se vislumbra la importancia de la investigación pedagógica dentro del proceso de formación de maestros y el matiz particular dado en cada línea de investigación, esta importancia a su vez ha permitido potenciar nuestro ser y quehacer a través del desarrollo de proyectos semestrales que pretenden articular la cotidianidad de la escuela y lo vivo desde los campos humanístico, científico, y cultural, aportando significativamente a los sujetos, los escenarios y las dinámicas por donde el maestro en formación se desenvuelve.

A partir del proceso de formación como Licenciados en Biología que hemos venido y seguiremos construyendo, se fomentan a diario un sinnúmero de vivencias propias y colectivas como el acercamiento a la escuela, los trayectos de la práctica pedagógica, el curso de algunos componentes, la vinculación a grupos, líneas y semilleros de investigación, esbozadas como experiencias nacientes desde diferentes lentes que impiden que la investigación se encasille en la homogenización y uniformidad del pensamiento y que por el contrario, alimentan la investigación desde diferentes cimientos, aristas y experiencias singulares, por lo tanto, la investigación es condición de posibilidad para diferentes miradas, en tanto que desde sus modos de constitución particular, intentan pensar de otro modo el maestro y la Biología y desde luego, el cruce de miradas de los encuentros, desencuentros y reflexiones que emanan a partir de los mismos.

En este sentido, la investigación pedagógica ha sido pensada a partir de múltiples formas que confluyen en la movilización del pensamiento de los sujetos que son participes de ella, en el caso del Departamento de Biología son experiencias que se vislumbran desde las socializaciones de la práctica pedagógica, así como también desde los conversatorios, los paneles, las ponencias y muestras en el marco de los tres encuentros realizados en los últimos años por el Departamento, los cuales han involucrado maestros y estudiantes tanto de Bogotá y de las extensiones (Chorrera, Valle de Tenza) como de otras universidades y/o instituciones educativas, haciendo posible no solo la divulgación y discusión en torno a sus hallazgos, sino también los cuestionamientos y las reflexiones que el trayecto investigativo trajo consigo, es así que se posibilita un diálogo de saberes con un modo particular de mostrarlo (audiovisuales, pósters, colecciones, fotografías...), desde la experiencia investigativa y la mirada particular en torno a los sujetos, objetos, escenarios y dinámicas que la constituyen. Por ello, considero que tanto compartir la experiencia 
investigativa, como contemplar la del otro, incita a ampliar la mirada sobre nuestro ser y quehacer, y sobre nosotros mismos, así como sobre aquello que nos genera inquietud o incluso no nos hemos preguntado, estas socializaciones nos han mostrado desde diferentes ópticas el matiz creador del maestro a la luz y la sombra de la investigación.

Así mismo, los trabajos de grado desarrollados al interior de las diferentes líneas de investigación, muestran pinceladas de creación al emanar desde las pasiones, afectos y experiencias que los estudiantes han tenido a lo largo de su formación, son trabajos que muestran un posicionamiento sobre la investigación tanto pedagógica como biológica de diversa índole, de manera que se pueden hilar y tejer relaciones creadoras de diferentes compresiones tendientes a propiciar movilizaciones desde la inquietud y la interrogación hacia otros modos de decir, cuya fuente de inspiración gravita en torno a la experiencia investigativa singular. De ahí que los maestros en formación vislumbren la investigación como parte de su ser y quehacer y no como trazos fragmentados que se supeditan y propenden de la funcionalidad, la estructuración y la reafirmación de verdades.

A partir de lo anterior, la práctica pedagógica del maestro y todo aquello que la va configurando, posibilita un encuentro que desde diversos escenarios da lugar al mirarse a sí mismo y al otro, una experiencia de saber, en tanto encuentro de movilización del pensar y el sentir que trae consigo problematizaciones y cuestionamientos que si bien pasan por el aula también transcienden a ella; la práctica pedagógica compone y recompone la historia de vida del maestro como sujeto singular, es una experiencia constante que deviene de muchas otras y por ello, siempre se da de manera distinta, no se replica ni se reproduce en tanto es singular, en sus trayectos, la práctica permite que el maestro despliegue su intelecto hacia la creación de posibilidades que lo movilicen a pensar su práctica constantemente pero siempre de manera diferente, desde sus afectos, emociones, sensaciones y sentimientos en relación con las instituciones y/o escenarios, los sujetos y las dinámicas en las que se involucra.

De ahí que, la práctica pedagógica se convierta en una posibilidad de plasmar, repensar y recrear esas experiencias singulares que devienen junto con la investigación a través de la escritura, puesto que como lo plantea Ávila (2003), ésta propicia que dichas experiencias se expongan al ámbito de la crítica y la discusión; así mismo, la escritura es una invitación a mostrar la experiencia del maestro, bien sea porque está ahí muy cerca de tocar las fibras de su corazón, o porque ha representado un logro, un fracaso, una búsqueda, una incertidumbre permanente o en pocas palabras, un choque que le ha transformado y que se halla permeado por sus sentires, pasiones y afectos. En este sentido, la escritura es un matiz propio del maestro, se configura como parte de su voz, una voz como posibilidad de dibujar y desdibujar la experiencia y las oscilaciones que ésta misma ha producido, como posibilidad de irrumpir en el mundo a través de palabras que se condensan entre líneas, líneas que cuentan historias singulares y que son capaces de movilizar y atravesar al otro, aún cuando no sepamos quien sea ese otro.

La investigación pedagógica entonces, no solo toma forma a partir de la palabra sino desde la escritura, como lo muestran las diferentes publicaciones (revistas, libros, artículos) de universidades y otras instituciones tanto nacionales como internacionales, que abarcan el despliegue de problematizaciones en torno a lo pedagógico y disciplinar, constituyendo así un amplio campo de inquietud y hallazgos que emanan como nuevas fibras movilizadoras tendientes a evidenciar que los maestros como sujetos intelectuales tienen algo que decir alrededor de su experiencia de vida, incluyendo desde su práctica pedagógica hasta sus modos particulares de ser y habitar este mundo, por tanto, ese algo que decir, no necesariamente debe estar inmerso en reafirmar la lógica de la resolución de problemáticas, la mejora de la calidad de vida y el progreso de las naciones.

Es así que con lo planteado en este escrito, surge entonces la pregunta por nuestro quehacer, la inquietud por el maestro de Biología y las relaciones que entreteje con la investigación y la disciplina que le es propia, puesto que no solo la investigación se constituye como eje del maestro desde sus intersticios, sino como eje de su objeto disciplinar, en nuestro caso, una Biología que merece la opción de ser pensada desde múltiples 
perspectivas que trasciendan la estrategia, la herramienta o el instrumento en pro de su enseñanza, puesto que la enseñanza según Martínez (2003) se entiende como vía hacia el pensamiento, en ese sentido, deconstruye la urgencia misma del acto de enseñar la Biología en el marco del discurso que permanece como verdad inamovible, es así que la enseñanza como pensamiento permite desplegar y emancipar nuestra experiencia como maestros en torno a diferentes posibilidades que impliquen imaginar, crear, contemplar, construir y deconstruir la Biología desde nuestro ser y existir propio, es decir, desde nuestro cuerpo, desde el debate y la discusión con el otro, no solo en torno a lo que se ha dicho, sino tal vez en torno a aquello que aún está por decir, desde nuestra ciudad y demás escenarios tanto naturales como urbanos que no cesan de mostrarnos que la Biología es parte de nosotros y como parte de nuestra vida nos invita a sorprendernos, asombrarnos, apasionarnos y conmovernos sin límites por el fenómeno de lo vivo y las relaciones que de allí emergen con nuestro diario vivir.

Por lo tanto, un maestro que no piensa la Biología fuera del marco del discurso que la posibilita, obstruye y reduce, es un maestro que probablemente no se piensa a sí mismo como sujeto ético, estético y político en tanto no propicia la movilización de su pensar y sentir desde la interrogación de su propio quehacer, desde la investigación como espacio de inquietud constante que permite ensanchar la mirada hacia otras formas de pensar la Biología, trascender lo disciplinar y construir nuevas formas de tejer relaciones y comprender el objeto biológico, más allá de involucrar lo vivo con la vida desde un sentido utilitarista enfocado en la posibilidad de solucionar las problemáticas y conflictos ambientales que nos acogen, así como en el diseño de instrumentos, herramientas y estrategias en función del aprendizaje de conceptos biológicos, vale la pena repensar esta relación entre lo vivo y la vida vislumbrando otras fibras que posibiliten que lo vivo y la vida interactúen desde los mismos hilos que tejemos y nos configuran como parte de este mundo, en el que nuestra capacidad de raciocinio no nos hace ser más ni mejores, simplemente, nos hace ser y estar en tanto creadores de múltiples posibilidades de relacionarnos consigo mismos, con el otro y con lo otro, relaciones que provienen de diferentes miradas que no se tildan de buenas o malas, solo son miradas que emergen de la experiencia y que constituyen un abanico de posibilidades para repensar esas relaciones que nos mantienen vivos a través del tiempo y el espacio, a través de la vida.

Finalmente, la invitación tanto a los maestros en formación como en ejercicio gira en torno a la configuración de nuestro ser y quehacer propio, de manera que la investigación pedagógica pueda tomar otros matices provenientes de diferentes campos del saber que puedan brindar sus aportes sin discriminación alguna, suscitando un dialogo de saberes con movilización constante hacia la imaginación y la creación de hilos que nos permitan trascender el acto de enseñar a la enseñanza como vía hacia el pensamiento, que retomando a Martínez (2003), implica abrir un espacio para el arte, la estética y la ética donde se recompongan los procesos de subjetivación en dirección hacia la invención y el acontecimiento de nuevas formas y posibilidades de ser y habitar en el mundo, más no de nuevos sujetos que se ajusten a las lógicas que imperan actualmente en relación con ciudadanos flexibles capaces de solucionar problemas y enfrentarse a situaciones de incertidumbre en lo que acontece a diario.

\section{Algunas consideraciones finales...}

Este escrito, se constituye en una de las tantas opciones que intenta pensar en el maestro y su experiencia de vida desde la investigación pedagógica, en tanto deviene a partir de diferentes sujetos, objetos, escenarios y dinámicas, por ello, no busca establecer conclusiones, sino por el contrario, pretende dejar inconclusos muchos aspectos que quedan bien por decir o decirlo de un modo diferente, estas líneas, configuran un espacio abierto a la inquietud, a la interrogación y a la opción de mirarse a sí mismo desde su ser y quehacer propio, desde su tejido singular en la experiencia de vida que cada quien está constituyendo, no se trata de plasmar, perfilar o reivindicar un ideal de maestro, de investigación pedagógica o de objeto biológico, tan solo se trata de vislumbrar un abanico de formas y posibilidades que permiten crearse y recrearse bien sea desde este mundo o desde otros donde la imaginación, la sensibilidad y las formas de ser y estar, se maticen como ventanas de escape que dibuja y desdibuja el maestro en su ir y devenir, ventanas que no son moldes ni replicas de otras, sino que por el contrario, son tan singulares como 
el mismo sujeto maestro que las recrea, porque emergen de su experiencia, de sus pasiones, afectos, luchas e incertidumbres, por ello, considero que hablar de creación, imaginación y sensibilidad no implica hablar de ideales que se queden tan solo en la palabra como aliciente.

La creación es posibilidad de ser y estar en el mundo que propicia muchas otras, pero no es una posibilidad limitada y restringida a estructurar herramientas o estrategias supeditadas a los procesos y problemas tanto del aprendizaje como del sujeto que aprende, es una posibilidad que nos transforma en su paso y que cuesta pensar e incluso mirar, es una posibilidad no solo para el maestro sino para el otro, no en función del aprendizaje y el lugar del desempeño o la flexibilidad, puesto que emana desde los pliegues internos y externos que nos constituyen como sujetos singulares. Es por ello, que desde los lentes de la pedagogía que han propiciado las movilizaciones de mi pensar y sentir, escribo con gran afecto estas palabras que transcienden mi experiencia al poder plasmarlas, al tener la opción de pensar en lo invisible, en aquello que no es urgente ni importante porque no otorga recetas, no estructura caminos homogeneizantes, ni mucho menos busca solucionar problemáticas.

Por tanto, la investigación como experiencia para los maestros en formación del Departamento de Biología, es una experiencia de saber que nos permite crear otros mundos posibles donde se piensa lo impensado, se piensa de otros modos que trascienden al marco, la forma regular, y la superficie de lo vivido, la experiencia como saber nos invita a pensar en la investigación desde la posibilidad amorfa de sujetarnos y desujetarnos constantemente tanto de lo que somos, como de aquello que se nos presenta como verdad legitima, y en ese sentido, ser sujetos creadores desde nuestro estilo de vida singular, desde lo que nos apasiona o nos inquieta, desde lo que es visible pero también desde lo que nos cuesta visibilizar, produce oscilaciones del pensar y el sentir del sujeto, oscilaciones que tienen mucho que ver con nuestra forma de vida como maestros, con nuestro ser y quehacer en cuestión y que desde luego, trascienden e interrogan la forma de vislumbrar y limitar la investigación a un mero proceso que produce, replica e innova y que se halla mediatizado a encontrar respuestas y posibles soluciones a las problemáticas de la humanidad en su afán por producir y modificar acorde a las necesidades imperantes.

\section{REFERENCIAS BIBLIOGRÁFICAS}

Ávila, Rafael (2003). La investigación acción pedagógica experiencias y lecciones. Bogotá: Ediciones Antropos Ltda. $307 \mathrm{p}$.

Cerda, Hugo (2007). La investigación formativa en el aula, la pedagogía como investigación. Bogotá: Investigar Magisterio. 219 p.

Larrosa, Jorge (2003). La experiencia de la lectura: estudios sobre literatura y formación. México: Fondo de Cultura Económica. 678 p.

Martínez, Alberto (2003). La enseñanza como posibilidad de pensamiento. En: Pedagogía y Epistemología. Bogotá: Cooperativa Editorial Magisterio. 301 p.

Martínez, Alberto (2008, Julio). Conferencia Magistral del encuentro "Semana de Reflexión e Intercambio Docente". Rostros y Rastros del Maestro Contemporáneo. Buenos Aires: Ministerio de Educación del Gobierno. $16 \mathrm{p}$.

Martínez, Alberto (2010, Abril). Vida de maestro. Conferencia Universidad Pedagógica Nacional. Bogotá, Colombia.

Tamayo, Alfonso (2008). El Movimiento Pedagógico en Colombia (Un encuentro de los maestros con la Pedagogía). Revista Histedbr On-line, No. 24 pp. 102-113. 
Noguera, Carlos (2002). Movimiento pedagógico, investigación y políticas educativas. Veinte años del pedagógico. Bogotá: Editorial Delfín Ltda. 325 p.

Roa, Paola; Osorio, Angélica y otros (2009, diciembre). Línea de Investigación Trayectos y Aconteceres: Estudios del Ser y el Quehacer del Maestro desde La Pedagogía. Una Mirada a las Elaboraciones Teóricas. Vol.2 No.3. Extraído el 28 de Abril de 2010, de: http://www.pedagogica.edu.co/revistas/ojs/index.php/biografia/article/view/286/360. $26 \mathrm{p}$.

Universidad Pedagógica Nacional (2005). Programa Académico de Licenciatura en Biología. 8 p.

Zuluaga, Olga (2003). El florecimiento de las investigaciones pedagógicas. En: Pedagogía y epistemología. Bogotá: Editorial Magisterio grupo historia de la práctica pedagógica. 301 p. 